\title{
Existence of Long-Range Order in the Migdal Recursion Equations
}

\author{
P. M. Bleher ${ }^{1}$ and E. Žalys ${ }^{2}$ \\ ${ }^{1}$ Institute of Applied Mathematics, Academy of Sciences, Moscow, USSR \\ 2 Institute of Mathematics and Cybernetics, Academy of Sciences of Lithuania SSR, SU-232600 \\ Vilnius, USSR
}

\begin{abstract}
A modification of XY-model is introduced for which Migdal recursion equation are exact. High- and low-temperature fixed points of these equations are investigated. As a result the existence of long-range order at low temperature and its absence at high temperature are proved rigorously for the model under consideration in the case when dimension $d>2$.
\end{abstract}

\section{Introduction}

The renormalization group method is widely used in physical works on phase transitions (see for instance [1-6]). At the same time a rigorous application of the method has been possible only for a narrow class of models (see [7-11]). But the simplicity and generality of the main ideas underlying the renormalization group provide a strong motivation for using it as a tool for rigorous investigations.

In this paper we shall use the renormalization group method to prove the existence of long-range order at low temperatures in a modified $d$-dimensional XY-model, when $d>2$. This model was first discussed in the papers by Migdal ([5, $6]$ ) and was defined for all real values of the dimension $d$ in the following way.

Let at first $d \geqq 2$ and $v \geqq 2$ be integers. We shall consider the hierarchical sequence of cubes $V_{n}, n=0,1, \ldots$ in $d$-dimensional Euclidean space $\mathbb{R}^{d}$ :

$$
V_{n}=\left\{x=\left(x_{1}, \ldots, x_{d}\right) \in \mathbb{R}^{d}, 0 \leqq x_{i} \leqq v^{n} ; i=1, \ldots, d\right\} .
$$

For every $n$ the whole space $\mathbb{R}^{d}$ is covered by cubes $V_{n, a_{m}}$ which appear as shifts of the $V_{n}$ by integer vectors $a_{m}=m v^{n}, m \in \mathbb{Z}^{d}$ proportional to $v^{n}$. Clearly, two cubes $V_{n, a_{m}}, V_{n, a_{m}}, m \neq m^{\prime}$ either do not intersect or have a whole face in common.

Let us denote by $S$ the set of closed $(d-1)$-dimensional elementary faces on the lattice $\mathbb{Z}^{d}$. Each face $s \in S$ is defined by an integer vector $m \in \mathbb{Z}^{d}$ (the point on the face with least coordinates assuming that the lexicographical ordering is introduced) and by the number $i$ of the axis to which the face $s$ in orthogonal. Thus each face can be written as $s_{m, j}$ where we denote the configuration space of the functions 
defined on $S$ and taking values on the circle $S^{1}$, as

$$
\Phi=\{\varphi=\{-\pi<\varphi(s) \leqq \pi, s \in S\} .
$$

We call two faces $s, s^{\prime} \in S$ equivalent and write $s \sim s^{\prime}$, if there exist $n \geqq 0$ and cube $V_{n, a_{m}}, m \in \mathbb{Z}^{d}, a_{m}=m v^{n}$ such that $s$ and $s^{\prime}$ belong to the same face of this cube.

The configuration space of Migdal model $\Phi_{0}$ is a subspace of $\Phi$ consisting of the configurations $\varphi$ for which $\varphi(s)=\varphi\left(s^{\prime}\right)$ when $s \sim s^{\prime}$.

The Hamiltonian of Migdal model is defined by the equation

$$
H(\varphi)=-\sum_{s_{m, j} ; s_{m+\delta_{j}, j} \in S} \cos \left(\varphi\left(\mathrm{s}_{m, j}\right)-\varphi\left(\mathrm{s}_{m+\delta_{j}, j}\right)\right),
$$

where $\delta_{j}$ is a vector with coordinates $\delta_{j}^{i}, i=1, \ldots, d$ and $\delta_{j}^{i}$ is the Kronecker symbol.

It can be seen that for this model the interaction of opposite faces is the same as for the XY-model, while equivalent faces interact in the not-random way.

If $V_{n}$ is a finite volume we define the Hamiltonian as

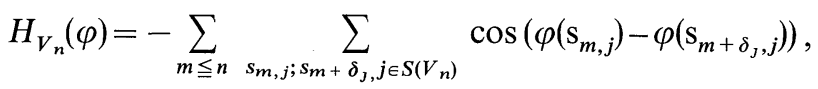

where $S\left(V_{n}\right)$ is the set of the faces of $S$ which lie in the volume $V_{n}$. The configuration spaces in the cube $V_{n}$ are

$$
\Phi\left(V_{n}\right)=\left\{\varphi=\left\{-\pi<\varphi(s) \leqq \pi, s \in V_{n} \cap S\right\}\right\}
$$

and

$$
\Phi_{0}\left(V_{n}\right)=\left\{\varphi \in \Phi\left(V_{n}\right) ; \varphi(s)=\varphi\left(s^{\prime}\right), \text { if } \quad s \sim s^{\prime}\right\} .
$$

We define the Gibbsian measure on $\Phi_{0}\left(V_{n}\right)$ corresponding to the Hamilton $H_{V_{n}}(\varphi)$ in the usual way

$$
\mu_{V_{n}}(d \varphi ; \beta)=\Xi_{V_{n}}^{-1}(\beta) \exp \left(-\beta H_{V_{n}}(\varphi)\right) \prod\left(\frac{d \varphi(s)}{2 \pi}\right),
$$

where the multiplication goes over classes of equivalent elements with respect to $\sim, \beta$ is the inverse temperature and the grand partition function in the volume $V_{n}$ is equal to

$$
\Xi_{V_{n}}(\beta)=\int \exp \left(-\beta H_{V_{n}}(\varphi)\right) \prod\left(\frac{d \varphi(s)}{2 \pi}\right) .
$$

We denote the boundary faces of cube $V_{n}$ by $s\left(V_{n}, j, \alpha\right)$, where $\alpha=1,2$ corresponds to upper and lower faces and $j=1, \ldots, d$ is the number of the axis orthogonal to $s$. By definition all the faces $s \in S$ which lie on the same face $s\left(V_{n}, j, \alpha\right)$ are equivalent, so that for each configuration $\varphi \in \Phi_{0}\left(V_{n}\right)$ all the values of $\varphi(s), s \in s\left(V_{n}, j, \alpha\right)$ are equal, i.e. $\varphi(s) \equiv \varphi_{j, \alpha}$. We shall denote by $G_{n}\left(x_{1,1}, x_{1,2}, \ldots, x_{d, 1}, x_{d, 2} ; \beta\right)$ the multidimensional probability distribution of the quantities $\varphi_{j, \alpha} ; \alpha=1,2 ; j=1, \ldots, d$ :

$$
G_{n}\left(x_{1,1}, \ldots, x_{d, 2} ; \beta\right)=\int \delta\left(\varphi_{1,1}-x_{1,1}\right) \ldots \delta\left(\varphi_{d, 2}-x_{d, 2}\right) \mu_{V_{n}}(d \varphi ; \beta) .
$$

The most important property of Migdal model is the existence of recurrence equations which relate $G_{n}$ and $G_{n+1}$. To obtain these relations we fix the values of 
configurations $\varphi \in \Phi_{0}\left(V_{n+1}\right)$ on the faces of cubes $V_{n, a_{m}} \subset V_{n+1}$ (i.e. on the faces of the $n$-th level)and consider their joint distribution

$$
\bar{G}_{n+1}\left(\left\{x_{s}, s \in \partial V_{n, a_{m}} ; V_{n, a_{m}} \subset V_{n+1}\right\} ; \beta\right)=\int \prod \delta\left(\varphi_{s}-x_{s}\right) \mu_{V_{n+1}}(d \varphi ; \beta),
$$

where $\partial V_{n, a_{m}}$ is the boundary of the cube $V_{n, a_{m}}$; the multiplication goes over all the faces $s \in \partial V_{n, a_{m}}\left(V_{n, a_{m}} \subset V_{n+1}\right)$ of $n$-th level, which lie in the volume $V_{n+1}$. As only opposite faces interact, it's easy to see that the following equation holds:

One can obtain the function $G_{n+1}$ from the $\bar{G}_{n+1}$ directly by integrating the variables $x_{s}$ where $s \in V_{n+1}$ but $s \bar{\in} \partial V_{n+1}$ :

$$
\begin{aligned}
& G_{n+1}\left(\left\{x_{s}, s \in \partial V_{n+1}\right\} ; \beta\right) \\
& \quad=\int \bar{G}_{n+1}\left(\left\{x_{s}, s \in \partial V_{n, a_{m}} ; V_{n, a_{m}} \subset V_{n+1}\right\} ; \beta\right) \prod_{s \in \bar{\partial} V_{n+1}}\left(\frac{d x_{s}}{2 \pi}\right) .
\end{aligned}
$$

Hence we have the desired relation:

$$
\begin{aligned}
& G_{n+1}\left(\left\{x_{s}, s \in \partial V_{n+1}\right\} ; \beta\right) \\
& \quad=\int \prod_{V_{n, a_{m}}} \prod_{V_{n+1}} G_{n}\left(\left\{x_{s}, s \in \partial V_{n, a_{m}}\right\} ; \beta\right) \prod_{s \in \partial V_{n+1}}\left(\frac{d x_{s}}{2 \pi}\right) .
\end{aligned}
$$

Presumably this equation can be obtained as a particular case of some general renormalization group transformation which is applicable also to translation invariant models (for this see [3]) but the exact definition of such a transformation is not yet known.

The initial condition for the recurrence relation (1.1) has the following form

$$
\begin{aligned}
G_{0}\left(\left\{x_{s}, s \in \partial V_{0}\right\} ; \beta\right) & =\Xi_{V_{0}}^{-1}(\beta) \exp \left(-\beta \sum_{j=1}^{d} \cos \left(x_{j, 1}-x_{j, 2}\right)\right) \\
& =(2 \pi)^{-d} \prod_{j=1}^{d} P_{0}\left(x_{j, 1}-x_{j, 2} ; \beta\right),
\end{aligned}
$$

where

$$
P_{0}(x ; \beta)=L_{0}^{-1} \exp (\beta \cos x) ; \quad L_{0}=\int_{-\pi}^{\pi} \exp (\beta \cos x) d x .
$$

It is evident that the function $G_{0}$ is written as a product of the functions $P_{0}$ which correspond to each of the $d$ coordinates. We shall show that all the functions $G_{n}$ can be represented as products of the corresponding functions $P_{n}$ :

$$
\begin{aligned}
G_{n}(x ; \beta)= & (2 \pi)^{-d} \prod_{j=1}^{d} P_{n}\left(x_{j, 1}-x_{j, 2} ; \beta\right) \\
& \int_{-\pi}^{\pi} P_{n}\left(x_{0} ; \beta\right) d x_{0}=1
\end{aligned}
$$

where $x=\left(x_{1,1}, \ldots, x_{d, 2}\right)$ and $P_{n}\left(x_{0}+2 \pi\right)=P_{n}\left(x_{0}\right)$ is a periodic function of $x_{0} \in \mathbb{R}^{1}$. This relation shows that spin variables corresponding to the faces of different directions are independent. We shall assume that this property is valid for the function $G_{n}$ and substitute (1.3) into the Eq. (1.1):

$$
G_{n+1}(x ; \beta)=\int \prod_{V_{n, a_{m}} \subset V_{n+1}}(2 \pi)^{-d} \prod_{j=1}^{d} P_{n}\left(x_{j, 1}^{\left(a_{m}\right)}-x_{j, 2}^{\left(a_{m}\right)} ; \beta\right) \prod_{s \in \partial V_{n+1}}\left(\frac{d x_{s}}{2 \pi}\right),
$$


where we denote by $x_{j, 1}^{\left(a_{m}\right)}$ (resp. $x_{j, 2}^{\left(a_{m}\right)}$ ) the spin variable on the lower (resp. upper) $j$-th face of the cube $V_{n, a_{m}}$ so that $x_{j, 1}^{\left(a_{m}+\delta_{j}\right)}$ is identified with $x_{j, 2}^{\left(a_{m}\right)}$.

It is easy to represent the last integral as the product of integrals corresponding to each direction. Moreover, it can be seen, that each such "directional integral" can be rewritten as the product of $v^{d-1}$ identical convolution type integrals

$$
\int_{-\pi}^{\pi} \ldots \int_{-\pi}^{\pi} \prod_{k=1}^{v-2} P_{n}\left(y_{k}-y_{k+1} ; \beta\right) d y_{k}=\left(P_{n} * \ldots * P_{n}\right)\left(x_{j, 1}-x_{j, 2} ; \beta\right) \text {. }
$$

As a result we obtain that

$$
G_{n+1}(x ; \beta)=(2 \pi)^{-d} \prod_{j=1}^{d} P_{n+1}\left(x_{j, 1}-x_{j, 2} ; \beta\right),
$$

where

$$
\begin{aligned}
& P_{n+1}(x ; \beta)=L_{n+1}^{-1}(\beta)(\underbrace{P_{n} * \ldots * P_{n}}_{\nu})^{v^{d-1}}(x ; \beta), \\
& L_{n+1}(\beta)=\int_{-\pi}^{\pi}\left(P_{n} * \ldots * P_{n}\right)^{v^{d-1}}(x ; \beta) d x .
\end{aligned}
$$

Let's now expand each of the functions $P_{n}(x ; \beta)$ in Fourier series with respect to $x$ in the interval $[-\pi, \pi]$

$$
P_{n}(x ; \beta)=(2 \pi)^{-1} \sum_{j=-\infty}^{\infty} \tilde{P}_{n}(j ; \beta) \exp (i j x) .
$$

Then according to (1.4), (1.5) we have

$$
\begin{aligned}
\tilde{P}_{n+1}(j ; \beta) & =\tilde{L}_{n+1}^{-1}(\beta) \underbrace{\left(\tilde{P}_{n}^{v} * \ldots * \tilde{P}_{n}^{v}\right)}_{v^{d}-1}(j ; \beta), \\
\tilde{L}_{n+1}(\beta) & =\left(\tilde{P}_{n}^{v} * \ldots * \tilde{P}_{n}^{v}\right)(0 ; \beta) .
\end{aligned}
$$

Given $F_{n}(j ; \beta)=\left(\tilde{P}_{n}(j ; \beta)\right)^{v}$ and $m=v^{d-1}$ these relations can be written as

$$
\begin{aligned}
& \left.Z_{n+1}(j ; \beta)=(\underbrace{\left(F_{n} * \ldots * F_{n}\right.}_{m})(j ; \beta)\right)^{v}, \\
& F_{n+1}(j ; \beta)=Z_{n+1}(j ; \beta) Z_{n+1}^{-1}(0 ; \beta) .
\end{aligned}
$$

One can choose the initial conditions for these recursion equations as

$$
Z_{-1}(j ; \beta)=(2 \pi)^{-1} \int_{-\pi}^{\pi} \exp \left(\frac{1}{m} \beta \cos x+i j x\right) d x=I_{j}\left(\frac{1}{m} \beta\right),
$$

where $I_{j}(z)$ is the modified Bessel function (see [12]). In such a case in fact

or

$$
F_{-1}(j ; \beta)=Z_{-1}(j ; \beta) Z_{-1}^{-1}(0 ; \beta) ; Z_{0}=\left(F_{-1} * \ldots * F_{-1}\right)^{m} \frac{1}{d-1}
$$

$$
P_{0}(x ; \beta)=\text { const } \cdot\left(\tilde{Z}_{-1}\right)^{m}=\text { const } \cdot \exp (\beta \cos x) ; \int_{-\pi}^{\pi} P_{0}(x ; \beta) d x=1
$$

in agreement with (1.2). For convenience in the sequel we shall write $Z_{n+1}, F_{n+1}$ instead of $Z_{n}, F_{n}, n=0,1, \ldots$ and rewrite the initial conditions as

$$
Z_{0}(j ; \beta)=(2 \pi)^{-1} \int_{-\pi}^{\pi} \exp \left(\frac{1}{m} \beta \cos x+i j x\right) d x=I_{j}\left(\frac{1}{m} \beta\right) .
$$


It is noteworthy that the Eqs. (1.6)-(1.8) make sense for all the integers $m \geqq 2$ and for any real value of the dimension $d$. Initially they were introduced in paper [6] as approximate equations for the usual XY-model. As far as our model concerned they are exact when $d$ and $v$ are integers. In the sequel we shall regard these equations as the definition of the model discussed in this paper. The same equations occur also in the gauge lattice models (see e.g. [5] and [18]).

The main purpose of this paper is the investigation of long-range order in the Eqs. (1.6)-(1.8). More precisely, we prove that these iterations behave differently when $n \rightarrow \infty$ at high and low temperatures.

In the sequel we shall assume that $m \geqq 2, d>2$ are integer and real fixed numbers respectively, otherwise arbitrary. It is possible to show that the functions $F_{n}(j ; \beta)$ are positive definite in the argument $j$, so that $F_{n}(j ; \beta) \leqq F_{n}(0 ; \beta)=1$ for all $j$.

As usual we denote with $\|\cdot\|_{l_{1}}$ the norm in the space $l_{1}$

$$
\|g(k)\|_{l_{1}}=\sum_{k=-\infty}^{\infty}|g(k)| \text {. }
$$

Let $\delta(j)$ be the function concentrated at the origin:

$$
\delta(j)= \begin{cases}1, & j=0 \\ 0, & j \neq 0\end{cases}
$$

The following two theorems are the main results of this paper.

Theorem 1. Let $d>2,0<\beta \leqq \frac{m}{4} \exp \left(-\left(\frac{v}{v-1}\right)^{2}\right)$. Then the functions $F_{n}(j ; \beta)$ satisfy the estimate

$$
\left\|F_{n}(j ; \beta)-\delta(j)\right\|_{l_{1}} \leqq A_{1} \exp \left(-A_{2} \exp \left(A_{3} n\right)\right),
$$

where

$$
A_{1}=\exp \left(-\frac{v}{v-1}\right) ; A_{2}=v^{-1}\left|\ln \left(\frac{4}{m} \beta\right)\right| ; A_{3}=\ln v ; v=m^{\frac{1}{d-1}} .
$$

Theorem 2. There exists $\beta_{0}>0$ such that for all $\beta>\beta_{0}$ there exists a sequence of positive numbers $\gamma_{1}(\beta), \gamma_{2}(\beta), \ldots$ tending to zero such that

$$
\left|F_{n}\left(x / \gamma_{n} ; \beta\right)-\exp \left(-x^{2}\right)\right|<\exp (-0,3(\ln \beta+n)) .
$$

Besides, when $n \rightarrow \infty$

$$
\gamma_{n} \cdot\left(\frac{v}{2}\right)^{-n / 2} \rightarrow c(\beta)
$$

where $c(\beta) \sqrt{2 \beta} \rightarrow 1$ when $\beta \rightarrow \infty$.

Thus by Theorem 1 the functions $F_{n}(j ; \beta)$ tend "double exponentially" to a $\delta$-function when $\beta$ is small enough, while by Theorem 2 for large $\beta$ the functions $F_{n}(j ; \beta)$ tend to 1 on every compact with exponential rate. The limit functions $\delta$-function and $\mathbf{1}$ - are high and low temperature fixed points of the transformations (1.6), (1.7). We need to note that these transformations are not defined for $F_{n} \equiv \mathbf{1}$ but it will be shown during the proof of Theorem 2 that if the function $F_{n}$ is close to $\mathbf{1}$ in an appropriate sence then the function $F_{n+1}$ is closer to $\mathbf{1}$ then $F_{n}$ is. 
At low temperatures it is natural to introduce the renormalized functions $f_{n}(x ; \beta)=F_{n}\left(x / \gamma_{n} ; \beta\right)$ which tend to $\exp \left(-x^{2}\right)$ when $n \rightarrow \infty$ according to Theorem 2 .

The Hamiltonian of the model under consideration is ferromagnetic for integer values of $d$ and $v\left(v=m^{1 / d-1}\right)$ so the Griffiths inequalites hold for it (see [14]). From these inequalities it follows the monotonicity of Fourier coefficients $F_{n}(j, \beta)$ in $\beta$ for fixed $j \neq 0$ and $n$. Apparently this property is also valid for all real values of $d$ and $v$ (e.g. this was discussed for $m=2$ and some values $d>2$ in the paper [15]). Using Griffiths inequalities it is possible to show the existence of weak limit of Gibbsian measures $\lim _{n \rightarrow \infty} \mu_{n}=\mu_{\infty}$, to prove the following estimate of binary correlation function

$$
\left\langle\cos \left(\varphi(s)-\varphi\left(s^{\prime}\right)\right)\right\rangle_{\mu_{\infty}} \geqq\left(\prod_{n=0}^{\infty} F_{n}(1 ; \beta)\right)^{v-1}
$$

for all $s, s^{\prime} \in S$ and to obtain some useful corollaries of Theorems 1 and 2 . In particular it follows from Theorem 2 , that for sufficiently low temperatures the quantity $1-F_{n}(1 ; \beta)$ is exponentially small in $n$, so

$$
\left(\prod_{n=0}^{\infty} F_{n}(1 ; \beta)\right)^{v-1}=m>0
$$

i.e. the values of binary correlation function are strictly positive, which means the existence of long-range order in this model. The other general result which is valid for the model under consideration is the Lee-Yang theorem (see [16]). Using it one can prove the absence of phase transitions for non-zero external field $h$ and, by Theorem 2, show the existence of a jump in the magnetization for $h=0$ and low temperatures. This means that the phase transition is of first order in $h$.

However it is necessary to point out that both the Lee-Yang theorem and the Griffiths inequalities are applicable in our model only when $d$ and $v$ are integers.

The critical point of the model: $T=T_{\mathrm{cr}}, h=0$ was considered in the paper [6] using perturbation theory and numerically in [15]. The main conclusion of the last paper was that the critical temperature tends presumably to zero when $d \rightarrow 2$ (the calculations were performed for $m=2$ ).

The idea of the proof of the Theorems 1 and 2 consists in a detailed investigation of the non-linear transformations (1.6), (1.7) in a neighborhood of the high- and low-temperature fixed points.

The reader can easily find that the proof of Theorem 2 involves some technical ideas of the papers [7-11]. During the proof we discuss the case $m=2$ only. More general case can be treated by obvious modifications.

We shall prove Theorem 1 in Sect. 2. The proof of Theorem 2 is more complicated and it will be discussed in Sects. 3-5.

\section{High Temperatures}

Proof of Theorem 1. At first we rewrite the function $F_{n}(j ; \beta)$ in the following form

$$
F_{n}(j ; \beta)=\delta(j)+h_{n}(j ; \beta)
$$

where $h_{n}(j ; \beta)$ is an even function of $j$, and $h_{n}(0 ; \beta)=0$ because it follows from the definition that $F_{n}(0 ; \beta)=1$. Then the substitution of (2.1) into the formula (1.6) 
(recall that $m=2$ ) gives:

$$
Z_{n+1}=\left(F_{n} * F_{n}\right)^{v}=\left(\delta+2 h_{n}+h_{n} * h_{n}\right)^{v} .
$$

Now we formulate two inequalities from which we shall derive Theorem 1:

$$
\begin{gathered}
\|h * h\|_{l_{1}} \leqq\|h\|_{l_{1}}^{2}, \\
\left\||h|^{v}\right\|_{l_{1}} \leqq\|h\|_{l_{1}}^{v} .
\end{gathered}
$$

The first one is widely-known, the second one is obtained in a simple way:

$$
\sum_{j=-\infty}^{\infty}|h(j)|^{v} \leqq\left|\sup _{j} h(j)\right|^{v-1} \sum_{j=\infty}^{\infty}|h(j)| \leqq\|h\|_{l_{1}}^{\nu-1}\|h\|_{l_{1}} .
$$

Let us denote

$$
Z_{n+1}(j ; \beta)=Z_{n+1}(0 ; \beta) \delta(j)+\tilde{h}_{n+1}(j ; \beta) ; \tilde{h}_{n+1}(0 ; \beta)=0 .
$$

It is evident that

$$
\left\|\tilde{h}_{n+1}\right\|_{l_{1}} \leqq\left\|2 h_{n}+h_{n} * h_{n}\right\|_{l_{1}}^{v} \leqq\left(2\left\|h_{n}\right\|_{l_{1}}+\left\|h_{n}\right\|_{l_{1}}^{2}\right)^{v} .
$$

Moreover in view of $h_{n} * h_{n}(0 ; \beta) \geqq 0$ the following estimate is true

$$
Z_{n+1}(0 ; \beta)=\left(1+h_{n} * h_{n}(0 ; \beta)\right)^{v} \geqq 1 .
$$

Hence for the function $h_{n+1}(j ; \beta)$ which is defined by the relations

$$
\begin{aligned}
& F_{n+1}(j ; \beta)=Z_{n+1}(j ; \beta) Z_{n+1}^{-1}(0 ; \beta)=\delta(j)+h_{n+1}(j ; \beta), \\
& h_{n+1}(j ; \beta)=\tilde{h}_{n+1}(j ; \beta) Z_{n+1}^{-1}(0 ; \beta)
\end{aligned}
$$

we have the estimate

$$
\left\|h_{n+1}\right\|_{l_{1}} \leqq\left\|\tilde{h}_{n+1}\right\|_{l_{1}} \leqq\left(2\left\|h_{n}\right\|_{l_{1}}+\left\|h_{n}\right\|_{l_{1}}^{2}\right)^{v},
$$

which gives the possibility of proving our theorem by induction. According to (1.8), when $m=2, Z_{0}(j ; \beta)=I_{j}\left(\frac{1}{2} \beta\right)$. Using the properties of the modified Bessel functions (see e.g. [12]) we easily obtain that

$$
\begin{aligned}
& Z_{0}(j ; \beta)=\sum_{k=0}^{\infty} \frac{\left(\frac{1}{4} \beta\right)^{2 k+j}}{k !(k+j) !}>0 \\
& Z_{0}(j ; \beta)=Z_{0}(-j ; \beta), Z_{0}(0 ; \beta)>1, \sum_{j=-\infty}^{\infty} Z_{0}(j ; \beta)=e^{\beta / 2} .
\end{aligned}
$$

Hence

$$
\left\|h_{0}\right\|_{l_{1}}=Z_{0}^{-1}(0 ; \beta) \sum_{j \neq 0} Z_{0}(j ; \beta)<e^{\beta / 2}-1<2 \beta .
$$

The last inequality is valid for all the values of $\beta$ which satisfy the condition of Theorem 1, which guarantes the initial step of the induction proof. Assume now, that

$$
\left\|h_{n}\right\|_{l_{1}} \leqq \exp \left(-C_{n}\right), \quad C_{n}>1
$$

Then

$$
\left\|h_{n+1}\right\|_{l_{1}} \leqq\left(\left(2+e^{-1}\right)\left\|h_{n}\right\|_{l_{1}}\right)^{v} \leqq \exp \left(-C_{n+1}\right),
$$


where $C_{n+1}=v C_{n}-v$. The solution of this recurrent equation is

$$
C_{n}=\frac{v}{v-1}+\left(C_{0}-\frac{v}{v-1}\right) v_{n} \text {. }
$$

As we have seen above it is possible to take $C_{0}=|\ln (2 \beta)|$. It follows from the condition of Theorem 1 that

$$
|\ln (2 \beta)|-\frac{v}{v-1}>v^{-1}|\ln (2 \beta)|
$$

so due to the last inequality

$$
C_{n}>v^{n-1}|\ln (2 \beta)|+\frac{v}{v-1}
$$

what completes the proof of Theorem 1.

\section{Low Temperatures}

We shall prove Theorem 2 also by induction. First we formulate some inductive assumptions for the functions $F_{n}(j ; \beta)$ and show that if they are valid for $n=n_{0}$, then they are valid also for $n=n_{0}+m$ where $m=m\left(n_{0}\right)=\left[\omega\left(\ln \beta+n_{0}\right)\right], \omega=10^{-3}$. After that we verify these assumptions for the initial function $F_{0}(j ; \beta)$. As a result they hold for the sequence of numbers $n_{0}=0, n_{1}, n_{2}, \ldots ; n_{i+1}=n_{i}+m\left(n_{i}\right)$. Finally we consider the behaviour of the functions $F_{n}(j ; \beta)$ for the intermediate values $n ; n_{i} \leqq n$ $<n_{i+1}$. In this section we give the exact formulations of the inductive assumptions and main lemmas and derive Theorem 2 from the lemmas.

Let us start with some notations. Let $n$ be fixed and $\gamma_{n}, 0<\gamma_{n}<1$ be a real number. It is useful to represent the function $F_{n}(j ; \beta)$ in the following renormalized form (in the sequel for brevity the dependence of $\beta$ is not indicated):

$$
F_{n}(j)=L_{n} \exp \left(-\left(\gamma_{n} j\right)^{2}\right)\left(1+r_{n}\left(y_{n} j\right), \quad j \in \mathbb{Z}^{1},\right.
$$

where $r_{n}\left(\gamma_{n} j\right)$ is an even function. We consider below also the function $f_{n}(x)$ $=F_{n}\left(x / \gamma_{n}\right)$ and the corresponding representation for it:

$$
f_{n}(x)=L_{n} \exp \left(-x^{2}\right)\left(1+r_{n}(x)\right),
$$

where the variable $x$ belongs to lattice $\mathscr{X}=\left\{x=k \Delta_{n}, k \in \mathbb{Z}^{1}\right\} \subset \mathbb{R}^{1}$, the lattice step $\Delta_{n}$ of which is equal to $\gamma_{n}$. Let us denote

$$
X_{n}(j)=\sum_{k=-\infty}^{\infty} F_{n}(k) F_{n}(j-k) .
$$

Then

$$
Z_{n+1}(j)=X_{n}^{v}(j) ; Z_{n+1}(0)=X_{n}^{v}(0) ; F_{n+1}(j)=\left(\frac{X_{n}(j)}{X_{n}(0)}\right)^{v} \text {. }
$$

Since $F_{n}(j)=f_{n}\left(\gamma_{n} j\right)$, given $t=k \gamma_{n}$, we obtain, that

$$
\begin{aligned}
X_{n}(j)= & \tilde{L}_{n} \exp \left(-\frac{1}{2}\left(\gamma_{n} j\right)^{2}\right) \sum_{t=-\infty}^{\infty} \exp \left(-2\left(\frac{1}{2} \gamma_{n} j-t\right)^{2}\right) \\
& \cdot\left(1+r_{n}(t)\right)\left(1+r_{n}\left(\gamma_{n} j-t\right)\right) \Delta_{n} .
\end{aligned}
$$


So according to (3.4)

$$
\begin{aligned}
F_{n+1}(j)= & L_{n+1}^{(1)} \exp \left(-\left(\gamma_{n} j \sqrt{\frac{v}{2}}\right)^{2}\right) \\
& \cdot\left(\sum_{t=-\infty}^{\infty} \exp \left(-2\left(\frac{1}{2} \gamma_{n} j-t\right)^{2}\right)\left(1+r_{n}(t)\right)\left(1+r_{n}\left(\gamma_{n} j-t\right)\right) \Delta_{n}\right)^{\nu},
\end{aligned}
$$

where $L_{n+1}^{(1)}=\tilde{L}_{n} Z_{n+1}^{-1}(0)$. Denoting $\gamma_{n+1}=\sqrt{\frac{\gamma}{2}} ; x=\gamma_{n+1} j ; f_{n+1}(x)=F_{n+1}\left(x / \gamma_{n}\right)$ we come to the relation

$$
f_{n+1}(x)=L_{n+1}\left(\sum_{t=-\infty}^{\infty} f_{n}\left(x \sqrt{\frac{2}{v}}-t\right) f_{n}(t) \Delta_{n}\right)^{v}
$$

or

$$
f_{n+1}(x)=L_{n+1} \exp \left(-x^{2}\right)\left(1+r_{n+1}(x)\right)
$$

where

$$
1+r_{n+1}(x)=\left(\sum_{t=-\infty}^{\infty} \exp \left(-2\left(\frac{x}{\sqrt{2 v}}-t\right)^{2}\right)\left(1+r_{n}(t)\right)\left(1+r_{n}\left(x \sqrt{\frac{2}{v}}-t\right)\right) \Delta_{n}\right)^{v} .
$$

The set $\left\{x:|x|<D_{n}=D \sqrt{\ln \beta+n}\right\}$ will be considered as the one of typical values of the variable $x$ (here $D$ is an absolute constant; for example, all the subsequent considerations are valid for $D=10$ ). Equation (3.7) will be fundamental for the estimate of the error $r_{n}(x)$ on the set of typical values.

Now let us formulate the inductive assumptions on the function $F_{n}(j)$. Denote

$$
A_{n}=\prod_{m=1}^{n}\left(1+\frac{1}{\sqrt{\beta}}\left(\frac{v}{2}\right)^{0.9 m}\right)^{-1}>1-\frac{\text { const }}{\sqrt{\beta}}
$$

and introduce the auxiliary function $\left(x_{n}>0\right)$

$$
\mathscr{F}_{n}\left(x, x_{n}\right)=\left\{\begin{array}{l}
1 / 2 \exp \left(-0.99 A_{n} x^{2}\right),|x|<x_{n} \\
\exp \left(-1 / 2 x_{n}^{2}-\left(|x| \ln |x|-x_{n} \ln x_{n}\right)\right),|x| \geqq x_{n}
\end{array}\right.
$$

Condition $\mathbf{V}^{(\boldsymbol{n})}$. There exist constants $L_{n}>0$ and $\gamma_{n}, 0<\gamma_{n}<\frac{1}{\sqrt{\beta}}\left(\frac{v}{2}\right)^{0.9 n}$ such that the function $f_{n}(x)=F_{n}\left(x / \gamma_{n}\right)$ can be written in the form (3.2) with the estimates:

$$
\left.\mathrm{v}_{1}\right)\left|r_{n}(x)\right| \leqq \delta_{n}=\frac{1}{\sqrt{\beta}}(2 v)^{-(1-\omega) n}, \quad \omega=10^{-3}
$$

when $x$ belongs to the set of typical values, i.e. $|x|<D_{n}$

$$
\left.\mathrm{v}_{2}\right)\left|f_{n}(x)\right| \leqq \mathscr{F}_{n}\left(x, 10 D_{n}\right), \quad \text { for } \quad|x| \geqq D_{n} .
$$


For the functions which satisfy the condition $\mathbf{V}^{(n)}$ we shall formulate three lemmas, from which Theorem 2 follows directly. We note that all our assertions in these lemmas should begin with the phrase: "There exists an inverse temperature $\beta_{0}>0$ such that for all $\beta>\beta_{0} \ldots$."

It means that all lemmas of this section are valid only for sufficiently large $\beta$.

Lemma 3.1. Condition $\mathbf{V}^{(n)}$ implies $\mathbf{V}^{(n+m)}$, for $m=m(n)=[\omega(\ln \beta+n)], \omega=10^{-3}$. Besides,

$$
\left|\gamma_{n+m} / \gamma_{n}\left(\frac{v}{2}\right)^{m / 2}-1\right|<\delta_{n}^{0.9}
$$

For any integer $p, 0 \leqq p<m=m(n)$ we shall consider the function $f_{n+p}(x)$ $=F_{n+p}\left(x / \gamma_{n+p}\right)$ where $\gamma_{n+p}=\left(\frac{v}{2}\right)^{p / 2} \gamma_{n}$. Now we represent it in the following form

$$
f_{n+p}(x)=L_{n+p} \exp \left(-x^{2}\right)\left(1+r_{n+p}(x)\right) \text {. }
$$

Lemma 3.2. If the condition $\mathbf{V}^{(n)}$ holds then the functions $f_{n+p}(x), 0 \leqq p<m=m(n)$ can be represented in the form (3.10) so that

$\left.\mathrm{p}_{1}\right)\left|r_{n+p}(x)\right| \leqq(2 v)^{(1+\omega) p} \delta_{n}$, for $|x|<D_{n+p}$;

$\mathrm{p}_{2}$ ) for $|x| \geqq D_{n+p}$ the inequality (3.9) is valid where $n$ is replaced by $n+p$.

Lemma 3.3. The initial function $F_{0}(j ; \beta)$ satisfies the condition $\mathbf{V}^{(0)}$ where $\gamma_{0}=(2 \beta)^{-1 / 2}$.

We shall prove these lemmas in $\S \S 4$ and 5. Now we obtain Theorem 2 as a consequence of Lemmas 3.1-3.3.

Proof of Theorem 2. From these lemmas it follows that the condition $\mathbf{V}^{(n)}$ holds for the sequence of numbers $n_{0}=0, n_{1}, n_{2}, \ldots$, which are defined by the recurrent relation $n_{i+1}=n_{i}+\left[\omega\left(\ln \beta+n_{i}\right)\right]$. It means that there exists a sequence of numbers $\gamma_{n_{i}}>0, i=1,2, \ldots$ such that

$$
F_{n_{i}}\left(x / \gamma_{n_{i}}\right) \equiv f_{n_{i}}(x)=L_{n_{i}} \exp \left(-x^{2}\right)\left(1+r_{n_{i}}(x)\right)
$$

where $L_{n_{i}}>0$ are some constants and all the functions $r_{n_{i}}(x)$ and $f_{n_{i}}(x)$ satisfy the estimates (3.8), (3.9) for $n=n_{i}$. Now define the quantities $\gamma_{n}$ and $\delta_{n}$ for the intermediate values $n ; n_{i} \leqq n<n_{i+1}$ as

$$
\gamma_{n}=\gamma_{n_{i}}\left(\frac{v}{2}\right)^{n-n_{i}} ; \quad \delta_{n}=\delta_{n_{i}}(2 v)^{(1+\omega)\left(n-n_{i}\right)} .
$$

Then combining both Lemmas 3.1 and 3.2 for all $n=0,1,2, \ldots$ we have the following representation

$$
F_{n}\left(x / \gamma_{n}\right) \equiv f_{n}(x)=L_{n} \exp \left(-x^{2}\right)\left(1+r_{n}(x)\right)
$$

in which $L_{n}>0$ are some constants and $r_{n}(x), f_{n}(x)$ satisfy the estimates (3.8), (3.9) respectively. Since $n-n_{i}<\omega(\ln \beta+n)$ it's easy to obtain for $\delta_{n}$ that

$$
\delta_{n}=\delta_{n_{i}}(2 v)^{(1+\omega)\left(n-n_{\imath}\right)}=\frac{(2 v)^{-(1-\omega) n}}{\sqrt{\beta}}(2 v)^{2\left(n-n_{i}\right)}<\frac{(2 v)^{-0.9 n}}{\beta^{1 / 3}} .
$$


In conclusion for any $n=0,1, \ldots$ the representation (3.11) holds with the estimates (3.12) and (3.9).

By definition $F_{n}(0)=1$, so $L_{n}=\left(1+r_{n}(0)\right)^{-1}$ and hence

$$
\left|L_{n}\left(1+r_{n}(x)\right)-1\right|=\frac{\left|r_{n}(x)-r_{n}(0)\right|}{1+r_{n}(0)}<3 \frac{(2 v)^{-0.9 n}}{\beta^{1 / 3}},|x|<D_{n} .
$$

Therefore, for $|x|<D_{n}$ we have

$$
\left|f_{n}(x)-\exp \left(-x^{2}\right)\right|=\exp \left(-x^{2}\right)\left|L_{n}\left(1+r_{n}(x)\right)-1\right|<\frac{(2 v)^{-0.9 n}}{\beta^{0.3}} .
$$

From (3.9) the same estimate is easily obtained for $|x| \geqq D_{n}$. Therefore the main part of Theorem 2 is proved. In conclusion we discuss the estimation of $\gamma_{n}$. By definition for all $n ; n_{i} \leqq n<n_{i+1}$ we have that $\gamma_{n}\left(\gamma_{n-1} \sqrt{\frac{v}{2}}\right)^{-1}=1$. According to Lemma 3.1 for $n=n_{i}$

$$
\left|\gamma_{n}\left(\gamma_{n-1} \sqrt{\frac{v}{2}}\right)^{-1}-1\right|<\delta_{n_{i}}^{0.9}<\delta_{n}^{0.8}
$$

i.e. for all $n=1,2, \ldots, \gamma_{n}\left(\gamma_{n-1} \sqrt{\frac{v}{2}}\right)^{-1}=1+\varepsilon_{n} ;\left|\varepsilon_{n}\right|<\delta_{n}^{0.8}$. Hence

$$
\frac{\gamma_{n}}{\gamma_{0}\left(\frac{v}{2}\right)^{n / 2}}=\prod_{k=1}^{n}\left(1+\varepsilon_{k}\right) \underset{n \rightarrow \infty}{\longrightarrow} \prod_{k=1}^{\infty}\left(1+\varepsilon_{k}\right)=c_{0}(\beta) .
$$

It is evident that $c_{0}(\beta) \rightarrow 1$ when $\beta \rightarrow \infty$. From Lemma 3.3 it follows that $\gamma_{0}=(2 \beta)^{-1 / 2}$. Therefore

$$
\frac{\gamma_{n}}{\left(\frac{v}{2}\right)^{n / 2}} \underset{n \rightarrow \infty}{\longrightarrow} \gamma_{0}(\beta) c_{0}(\beta)=c(\beta)
$$

and $c(\beta) \sqrt{2 \beta} \rightarrow 1$ when $\beta \rightarrow \infty$. Theorem 2 is proved.

\section{Proof of Lemmas 3.1 and 3.2}

The proof of these lemmas is carried out in the following way. First we estimate the function $r_{n+p}, p=1,2, \ldots, m$ on the set of typical values $\left\{|x|<D_{n+p}\right\}$ under the assumption that the external estimate $\mathrm{p}_{2}$ ) holds. The crucial point here is the proof of the inequality $v_{1}$ ) while the rough estimate $p_{1}$ ) of Lemma 3.2 is obtained rather easily. Next we prove the validity of the external estimate $v_{2}$ ) [and also $\left.p_{2}\right)$ ] for the function $f_{n+p}, 1 \leqq p \leqq m$ under the assumption that the function $f_{n+p-1}(x)$ satisfies the conditions of Lemma 3.2. One can see that combination of these results gives the proof of Lemmas 3.1 and 3.2. Indeed, first we prove Lemma 3.2 by induction and after that, using the external estimate obtained for $p=1, \ldots, m-1$ verify the 
inequality $\mathrm{v}_{1}$ ) for the error $r_{n+m}(x)$. In conclusion the external estimate $\mathrm{v}_{2}$ ) is proved.

It is noteworthy that almost all the considerations below are valid only for sufficiently large $\beta$, but as a rule we dont indicate the exact values of $\beta$ for which the estimates are satisfied. We would like to note also that when $v$ tends to 2 the estimate for $\beta$ becomes non-uniform in $v$. The last fact is completely natural because critical temperature tends presumably to 0 when $v \rightarrow 2$.

Rewrite the recurrence relation (3.5):

$$
\begin{aligned}
f_{n+1}(x)= & L_{n+1}\left(\sum _ { t \in T _ { n , x } } f _ { n } \left(x \sqrt{\left.\left.\frac{2}{v}-t\right) f_{n}(t) \Delta_{n}+R_{n+1}^{(1)}(x)\right)^{v}}\right.\right. \\
= & L_{n+1} \exp \left(-\frac{x^{2}}{v}\right)\left(\sum_{t \in T_{n, x}} \exp \left(-2\left(\frac{x}{\sqrt{2 v}}-t\right)^{2}\right)\right. \\
& \left.\cdot\left(1+2 r_{n}(t)+r_{n}(t) r_{n}\left(x \sqrt{\frac{2}{v}}-t\right)\right) \Delta_{n}+R_{n+1}^{(2)}(x)\right)^{v},
\end{aligned}
$$

where

$$
\begin{aligned}
T_{n, x}= & \left\{t:|t|,|x| \sqrt{\frac{2}{v}}-t \mid<D_{n}=D \sqrt{\ln \beta+n}\right\}, \\
& R_{n+1}^{(1)}(x)=\sum_{t \in T_{n, x}} f_{n}\left(x \sqrt{\frac{2}{v}}-t\right) f_{n}(t) \Delta_{n}, R_{n+1}^{(2)}(x)=\exp \left(\frac{x^{2}}{v}\right) R_{n+1}^{(1)}(x) .
\end{aligned}
$$

Using the external estimate (3.9) and inequality $\left|f_{n}(x)\right| \leqq 1$, we obtain that (here and further the symbols $c_{1}, c_{2}, \ldots$ denote some positive constants)

$$
\left|R_{n+1}^{(2)}(x)\right| \leqq\left|\tilde{R}_{n+1}^{(2)}(x)\right|+\left|\tilde{\tilde{R}}_{n+1}^{(2)}(x)\right|<\delta_{n}^{3}
$$

because for $|x|<D_{n+1}$

$$
\begin{aligned}
\left|\tilde{R}_{n+1}^{(2)}(x)\right|= & \left.\exp \left(\frac{x^{2}}{v}\right)\right|_{D_{n} \leqq|t|<10 D_{n}} f_{n}\left(x \sqrt{\frac{2}{v}}-t\right) f_{n}(t) \Delta_{n} \mid \\
& \leqq \frac{1}{4} \exp \left(\frac{x^{2}}{v}\right)_{\left|\frac{x}{\sqrt{2 v}}-t\right|>D_{n}\left(1+\frac{1}{\sqrt{2}}\right)} \exp \left(-1.97\left(\frac{x}{\sqrt{2 v}}-t\right)^{2}\right) \Delta_{n} \\
& <c_{1} e^{-D_{n+1}^{2}}<\frac{1}{2} \delta_{n}^{3}
\end{aligned}
$$

and

$$
\begin{aligned}
\left|\tilde{\tilde{R}}_{n+1}^{(2)}(x)\right|= & \exp \left(\frac{x^{2}}{v}\right)\left|\sum_{|t| \geqq 10 D_{n}} f_{n}\left(x \sqrt{\frac{2}{v}}-t\right) f_{n}(t) \Delta_{n}\right| \\
& <2 \exp \left(\frac{x^{2}}{v}\right) \sum_{|t| \geqq 10 D_{n}} f_{n}(t) \Delta_{n} \\
& <c_{2} \exp \left(\frac{1}{v} D_{n+1}^{2}-50 D_{n}^{2}\right)<\frac{1}{2} \delta_{n}^{3}
\end{aligned}
$$


Both the inequalities are evidently valid for $D=10$. The variables $t, x \sqrt{\frac{2}{v}}-t \in T_{n, x}$ belong to the set of typical values, therefore we can use inequality $v_{1}$ ) for an estimation of the errors $r_{n}(t)$ and $r_{n}\left(x \sqrt{\frac{2}{v}}-t\right)$. In what follows will be essential the property: if $|x|<D_{n+1}=D \sqrt{\ln \beta+n+1}$ and $\left|\frac{x}{\sqrt{2 v}}-t\right|<\frac{1}{4} D_{n}$ then $t \in T_{n, x}$. Indeed

$$
|t|,|x| \sqrt{\frac{2}{v}}-t|\leqq| \frac{x}{\sqrt{2 v}}-t|+| \frac{x}{\sqrt{2 v}} \mid \leqq \frac{1}{4} D_{n}+\frac{1}{\sqrt{2 v}} D_{n+1}<D_{n} .
$$

Taking this into account we have for $|x|<D_{n+1}$ that

$$
\begin{aligned}
& \sum_{t \in T_{n, x}} \exp \left(-2\left(\frac{x}{\sqrt{2 v}}-t\right)^{2}\right) \Delta_{n} \\
& \quad=\sum_{\left|\frac{x}{\sqrt{2 v}}-t\right|<\frac{1}{4} D_{n}} \exp \left(-2\left(\frac{x}{\sqrt{2 v}}-t\right)^{2}\right) \Delta_{n}+R_{n+1}^{(3)}(x),
\end{aligned}
$$

where

$$
R_{n+1}^{(3)}(x)=\sum_{\left|\frac{x}{\sqrt{2 v}}-t\right| \geqq \frac{1}{4} D_{n}} \exp \left(-2\left(\frac{x}{\sqrt{2 v}}-t\right)^{2}\right) \Delta_{n}=o\left(\exp \left(-\frac{1}{8} D_{n}^{2}\right)\right) .
$$

It is sufficient to take $D=10$ to bound $R_{n+1}^{(3)}(x)$ with $\delta_{n}^{3}$. Putting $u=\frac{x}{\sqrt{2 v}}-t$ we
have, that

$$
\sum_{|u|<\frac{1}{4} D_{n}} \exp \left(-2 u^{2}\right) \Delta_{n}=\int_{-\frac{1}{4} D_{n}}^{\frac{1}{4} D_{n}} \exp \left(-2 u^{2}\right) d u+R_{n+1}^{(4)}(x) .
$$

We shall show below [see (4.21)] that the error arising from the substitution of the sum by the integral can be bounded by $\delta_{n}^{3}$. Moreover, it is evident that

$$
\int_{-\frac{1}{4} D_{n}}^{\frac{1}{4} D_{n}} \exp \left(-2 u^{2}\right) d u=\sqrt{\frac{\pi}{2}}+R_{n+1}^{(5)}
$$

with an analogous estimate of the remainder term $R_{n+1}^{(5)}$.

Similarly one can obtain that

$$
\begin{aligned}
& \sum_{t \in T_{n}, x} r_{n}(t) \exp \left(-2\left(\frac{x}{\sqrt{2 v}}-t\right)^{2}\right) \Delta_{n} \\
& \quad=\sum_{|t|<D_{n}} r_{n}(t) \exp \left(-2\left(\frac{x}{\sqrt{2 v}}-t\right)^{2}\right) \Delta_{n}+R_{n+1}^{(6)}(x),
\end{aligned}
$$

where $\left|R_{n+1}^{(6)}(x)\right|<\delta_{n}^{3}$. For the last term of the Eq. (4.1) r.h.s., inequality $\mathrm{v}_{1}$ ) gives that

$$
\sum_{t \in T_{n, x}} r_{n}(t) r_{n}\left(x \sqrt{\frac{2}{v}}-t\right) \exp \left(-2\left(\frac{x}{\sqrt{2 v}}-t\right)^{2}\right) \Delta_{n}<\delta_{n}^{2}\left(\sqrt{\frac{\pi}{2}}+\delta_{n}^{3}\right) .
$$


Taking into account all the relations obtained above we have for $|x|<D_{n+1}$ that

$$
\begin{aligned}
f_{n+1}(x)= & L_{n+1} \exp \left(-\frac{x^{2}}{v}\right) \\
& \cdot\left(\sqrt{\frac{\pi}{2}}+2 \sum_{|t|<D_{n}} r_{n}(t) \exp \left(-2\left(\frac{x}{\sqrt{2 v}}-t\right)^{2}\right) \Delta_{n}+R_{n+1}^{(7)}(x)\right),
\end{aligned}
$$

where $\left|R_{n+1}^{(7)}(x)\right|<\delta_{n}^{2}\left(\sqrt{\frac{\pi}{2}}+5 \delta_{n}\right)$. Furthermore using the formula $(1+\alpha)^{v}=1+v \alpha$ $+o\left(\alpha^{2}\right)$ we can write the following representation

$$
f_{n+1}(x)=\bar{L}_{n+1} \exp \left(-x^{2}\right)\left(1+r_{n+1}(x)\right),
$$

where

$$
r_{n+1}(x)=2 v \sqrt{\frac{2}{\pi}} \sum_{|t|<D_{n}} r_{n}(t) \exp \left(-2\left(\frac{x}{\sqrt{2 v}}-t\right)^{2}\right) \Delta_{n}+\bar{R}_{n+1}(x)
$$

and

$$
\left|\bar{R}_{n+1}(x)\right|<5 \delta_{n}^{2} .
$$

Introducing the linear operator

$$
\mathbb{A}_{n} r_{n}(x)=2 v \sqrt{\frac{2}{\pi}} \sum_{|t|<D_{n}} r_{n}(t) \exp \left(-2\left(\frac{x}{\sqrt{2 v}}-t\right)^{2}\right) \Delta_{n}
$$

we rewrite (4.4) as

$$
r_{n+1}(x)=\mathbb{A}_{n} r_{n}(x)+\bar{R}_{n+1}(x) .
$$

Since

$$
\begin{aligned}
\left|\mathbb{A}_{n} r_{n}(x)\right| \leqq & 2 v \sqrt{\frac{2}{\pi}} \sup _{|t|<D_{n}}\left|r_{n}(t)\right| \sum_{|t|<D_{n}} \exp \left(-2\left(\frac{x}{\sqrt{2 v}}-t\right)^{2}\right) \Delta_{n} \\
& <2 v \delta_{n}\left(1+\delta_{n}\right)
\end{aligned}
$$

for $|x|<D_{n+1}$ it follows from (4.5) that

$$
\left|r_{n+1}(x)\right|<2 v \delta_{n}\left(1+\delta_{n}\right)+5 \delta_{n}^{2}<(2 v)^{1+\omega} \delta_{n} .
$$

Repeating these considerations $p$ times we shall obtain the estimate $p_{1}$ ) of Lemma 3.2 .

In connection with this estimate we indicate a useful inequality which is valid for all $p ; 0 \leqq p \leqq m$

$$
(2 v)^{(1+\omega) p}<5^{m}<\beta^{0.002}(2 v)^{0.003 n}<\delta_{n}^{-0.1}
$$

Let's go to the proof of the estimate $\mathrm{v}_{1}$ ) for the error $r_{n+p}(x)$. By (4.6) the function $q_{n+1}(x)=\mathbb{A}_{n} r_{n}(x)$ can be defined for all the real values $x$. Besides

$$
\left|\frac{d^{k}}{d x^{k}} q_{n+1}(x)\right|=2 v \sqrt{\frac{2}{\pi}} \sum_{|t|<D_{n}} r_{n}(t) \frac{d^{k}}{d x^{k}} \exp \left(-2\left(\frac{x}{\sqrt{2 v}}-t\right)^{2}\right) \Delta_{n} \mid<c_{3}^{(k)} \delta_{n}
$$


so

$$
\left\|q_{n+1}(x)\right\|_{C^{k}\left(\mathbb{R}^{1}\right)}<c_{4}^{(k)} \delta_{n}, \quad c_{4}^{(k)}=\sum_{0 \leqq l \leqq k} c_{3}^{(l)}
$$

for any $k=0,1, \ldots$ It's evident that the constants $c_{3}^{(k)}$ do not depend on both $\beta$ and $n$.

For $p=1, \ldots, m$ we use a recurrence relation which is analogous to (4.7):

$$
r_{n+p+1}(x)=\mathbb{A}_{n+p} r_{n+p}(x)+\bar{R}_{n+p+1}(x),
$$

where according to (4.5), (4.8), and $\mathrm{p}_{1}$ )

$$
\left|\bar{R}_{n+p+1}(x)\right|<5\left((2 v)^{(1+\omega) p} \delta_{n}\right)^{2}<\frac{1}{2} \delta_{n}^{1.8} .
$$

In the sequel it will be more convenient for us to introduce an integral operator instead of a difference operator. Namely we denote $r_{n+p}(x)=q_{n+p}(x)+R_{n+p}(x)$ and rewrite (4.10) as

$$
r_{n+p+1}(x)=q_{n+p+1}(x)+R_{n+p+1}(x),
$$

where

$$
\begin{aligned}
& q_{n+p+1}(x)=\mathscr{A} q_{n+p}(x) \equiv 2 v \sqrt{\frac{2}{\pi}} \int_{-\infty}^{\infty} q_{n+p}(t) \exp \left(-2\left(\frac{x}{\sqrt{2 v}}-t\right)^{2}\right) d t, \\
& R_{n+p+1}(x)=\bar{R}_{n+p+1}(x)+\mathbb{A}_{n+p} R_{n+p}(x)+\left(\mathbb{A}_{n+p}-\mathscr{A}\right) q_{n+p}(x) .
\end{aligned}
$$

The linear integral operator $\mathscr{A}$ is compact and selfadjoint in the Hilbert space (see e.g. [13])

$$
\begin{aligned}
\mathscr{H}(\gamma)= & L_{\mathrm{ev}}^{2}\left(\mathbb{R}^{1} ; \exp \left(-\gamma x^{2}\right)\right) \\
= & \left\{f(x):\|f(x)\|^{2}=\int_{-\infty}^{\infty}|f(x)|^{2} \exp \left(-\gamma x^{2}\right) d x<\infty ;\right. \\
& \left.f(x)=f(-x), x \in \mathbb{R}^{1}\right\}, \gamma=2-\frac{1}{v} .
\end{aligned}
$$

Its spectrum consists of the infinite sequence of numbers $2 v, 1,(2 v)^{-1}, \ldots$. The corresponding eigenfunctions are the even normed Hermite polynomials $G_{2 k}(x ; \gamma)$; $\left\|G_{2 k}(x ; \gamma)\right\|=1, k=0,1, \ldots$.

Now we expand the functions $q_{n+p}(x), p=1,2, \ldots$ in terms of Hermite polynominals $G_{2 k}(x ; \gamma)$ up to some large order $N$ :

$$
q_{n+p}(x)=\sum_{k=0}^{N} b_{n+p}^{(k)} G_{2 k}(x ; \gamma)+Q_{n+p}(x),
$$

where $Q_{n+p}(x)=\sum_{k=N+1}^{\infty} b_{n+p}^{(k)} G_{2 k}(x ; \gamma)$. The integer $N$ is an absolute constant which will be chosen below [see (4.37)]. It follows from (4.13) that

$$
\begin{aligned}
& b_{n+p+1}^{(k)}=(2 v)^{-(k-1)} b_{n+p}^{(k)}, \\
& \left\|Q_{n+p+1}(x)\right\|_{\mathscr{H}(\gamma)}=\left\|\mathscr{A} Q_{n+p}(x)\right\|_{\mathscr{H}(\gamma)} \leqq(2 v)^{-N}\left\|Q_{n+p}(x)\right\|_{\mathscr{H}(\gamma)} .
\end{aligned}
$$


To obtain the analogous recurrence estimate for $R_{n+p+1}(x)$ we note that for $|x|<D_{n+p+1}$

$$
\begin{aligned}
& \left|\mathbb{A}_{n+p} R_{n+p}(x)\right| \\
& \quad \leqq \sup _{|x|<D_{n+p}}\left|\mathrm{R}_{n+p}(x)\right| 2 v \sqrt{\frac{2}{\pi}} \sum_{|t|<D_{n}} \exp \left(-2\left(\frac{x}{\sqrt{2 v}}-t\right)^{2}\right) \Delta_{n} \\
& \quad<4 \sup _{|x|<D_{n+p}}\left|R_{n+p}(x)\right| .
\end{aligned}
$$

Besides

$$
\begin{aligned}
& \left(\mathbb{A}_{n+p}-\mathscr{A}\right) q_{n+p}(x) \\
& =2 v \sqrt{\frac{2}{\pi}}\left[\left(\sum_{|t|<D_{n+p}} \Delta_{n}-\int_{-D_{n+p}}^{D_{n+p}} d t\right) q_{n+p}(t) \exp \left(-2\left(\frac{x}{\sqrt{2 v}}-t\right)^{2}\right)\right. \\
& \left.\quad-\int_{|t| \geqq D_{n+p}} q_{n+p}(t) \exp \left(-2\left(\frac{x}{\sqrt{2 v}}-t\right)^{2}\right) d t\right] .
\end{aligned}
$$

For the estimation of the error arising from the substitution of the sum with the integral we use the following formula (see, e.g. [17])

$$
\int_{a}^{b} f(x) d x=\sum_{j=0}^{N} f(a+j \Delta) \Delta+R f, N \Delta=b-a
$$

in which

$$
|R f|<c_{5}^{(k)}\|f\|_{C^{k}([a, b])} \Delta^{k}+\sup _{a<x<a+k \Delta}|f(x)|+\sup _{b<x<b+k \Delta}|f(x)| .
$$

Hence we have that

$$
\begin{aligned}
\mathbb{J}= & \left|\left(\sum_{|t|<D_{n+p}} \Delta_{n}-\int_{|t|<D_{n+p}} d t\right) q_{n+p}(t) \exp \left(-2\left(\frac{x}{\sqrt{2 v}}-t\right)^{2}\right)\right| \\
& <c_{6}^{(k)}\left\|q_{n+p}(t) \exp \left(-2\left(\frac{x}{\sqrt{2 v}}-t\right)^{2}\right)\right\|_{C^{k}\left(\mathbb{R}^{1}\right)} \Delta_{n}^{k} \\
& +c_{7}\left\|q_{n+p}\right\|_{C\left(\mathbb{R}^{1}\right)} \exp \left(-2\left(\frac{x}{\sqrt{2 v}}-D_{n+p}\right)^{2}\right) .
\end{aligned}
$$

So for $|x|<D_{n+p+1}$

$$
\mathbb{J}<c_{8}^{(k)}\left\|q_{n+p}\right\|_{C^{k}\left(\mathbb{R}^{1}\right)} \Delta_{n+p}^{k}+c_{7} \exp \left(-D^{2}(\ln \beta+n+p)\right) .
$$

Because $\Delta_{n+p}=\gamma_{n+p}$ it follows from the condition $\mathbf{V}^{(n)}$ that

$$
\Delta_{n+p}<\beta^{-1 / 2}\left(\frac{v}{2}\right)^{0.45(n+p)} .
$$


As we can choose the constant $k$ sufficiently large ( $k$ depends only on $v$ ) taking $D=10$ we obtain that

$$
\mathrm{J}<c_{8}^{(k)}\left\|q_{n+p}\right\|_{C^{k\left(\mathbb{R}^{1}\right)}} \delta_{n}^{3}+c_{7} \delta_{n}^{3} .
$$

Since for each $p^{\prime}: 1 \leqq p^{\prime}<p$

$$
\left\|q_{n+p^{\prime}+1}\right\|_{C^{k}\left(\mathbb{R}^{1}\right)} \leqq \sup _{r \leqq k} \sup _{x}\left|\frac{d^{r}}{d x^{r}} \mathscr{A} q_{n+p^{\prime}}(x)\right| \leqq 4\left\|q_{n+p^{\prime}}(x)\right\|_{C^{k}\left(\mathbb{R}^{1}\right)}
$$

then taking into account (4.8) it follows from the inequality (4.9) that

$$
\left\|q_{n+p}(x)\right\|_{C^{k}\left(\mathbb{R}^{1}\right)} \leqq 4^{p} c_{9} \delta_{n}<\delta_{n}^{0.9} .
$$

In an analogous way it is proved that

$$
\left|\int_{|t| \geqq D_{n+p}} q_{n+p}(t) \exp \left(-2\left(\frac{x}{\sqrt{2 v}}-t\right)^{2}\right) d t\right|<\delta_{n}^{3} .
$$

Now from the inequalities (4.11) and (4.21)-(4.23) we directly obtain the recurrence estimation

$$
\left|R_{n+p+1}(x)\right|<4 \sup _{|x|<D_{n+p}}\left|R_{n+p}(x)\right|+\delta_{n}^{1.8} .
$$

From here and the inequalities (4.5) and (4.8) it is easy to deduce that

$$
\left|R_{n+p}(x)\right|<4^{p}\left(5 \delta_{n}^{2}+\delta_{n}^{1.8}\right)<\delta_{n}^{1.7} .
$$

Next using the (4.9) we obtain estimates of the quantities $b_{n+1}^{(k)}$ and $Q_{n+1}(x)$

$$
\left|b_{n+1}^{(k)}\right|,\left\|Q_{n+1}(x)\right\|_{\mathscr{H}(\gamma)} \leqq\left\|q_{n+1}(x)\right\|_{C\left(\mathbb{R}^{1}\right)} \sqrt{\int_{-\infty}^{\infty} \exp \left(-\gamma x^{2}\right) d x}<c_{10} \delta_{n} .
$$

Hence

$$
\begin{aligned}
& \left|b_{n+p}^{(k)}\right| \leqq c_{11} \delta_{n}(2 v)^{-(k-1)(p+1)}, \quad k=0, \ldots, N, \\
& \left\|Q_{n+p}(x)\right\|_{\mathscr{H}(\gamma)} \leqq c_{12} \delta_{n}(2 v)^{-N(p+1)} .
\end{aligned}
$$

The estimates (4.25)-(4.27) were obtained for an arbitrary $p=1, \ldots, m$. Now we use them for $p=m$. Namely

$$
\begin{aligned}
& f_{n+m}(x)=L_{n+m} \exp \left(-x^{2}\right)\left(1+r_{n+m}(x)\right) \\
& 1+r_{n+m}(x)=\sum_{k=0}^{N} b_{n+m}^{(k)} G_{2 k}(x ; \gamma)+Q_{n+m}(x)+R_{n+m}(x)
\end{aligned}
$$

with the estimates listed below

$$
\begin{aligned}
& \left|b_{n+m}^{(k)}\right|<c_{13}(2 v)^{-(k-1)(m+1)} \delta_{n}, \quad k=0, \ldots, N, \\
& \left\|Q_{n+m}(x)\right\|_{\mathscr{H}(\gamma)}<c_{14}(2 v)^{-N(m+1)} \delta_{n}, \\
& \sup _{|x|<D_{n+m}}\left|R_{n+m}(x)\right|<\delta_{n}^{1.7}<\delta_{n+m}^{1.6} .
\end{aligned}
$$


It is easily seen, that

$$
\begin{aligned}
\sup _{|x|}<D_{n+m} & \left|\sum_{k=2}^{N} b_{n+m}^{(k)} G_{2 k}(x ; \gamma)\right| \\
& <c_{15} \delta_{n} N(2 v)^{-(m+1)} \sup _{2 \leqq k \leqq N} \sup _{|x|<D_{n+m}}\left|G_{2 k}(x ; \gamma)\right| \\
& <c_{16} \delta_{n+m}(2 v)^{-(m+1)} D_{n+m}^{N},
\end{aligned}
$$

where $\delta_{n+m}=\beta^{-1 / 2}(2 v)^{-(1-\omega)(n+m)}$. Let $g=\ln \beta+n$. Then

$$
\begin{aligned}
& (2 v)^{-(m+1)}<(2 v)^{-\omega(\ln \beta+n)}<2^{-\omega g}, \\
& D_{n+m}^{N}=D^{N}(\ln \beta+n+m)^{N / 2}<c_{17} g^{N / 2},
\end{aligned}
$$

where $c_{17}$ does not depend on $\beta$ and $n$. Hence it follows that

$$
c_{16} \delta_{n+m}(2 v)^{-(m+1)} D_{n+m}^{N}<c_{16} \delta_{n+m} 2^{-\omega g} g^{N / 2}<\frac{1}{3} \delta_{n+m}
$$

when $\beta$ (and consequently $g$ ) is sufficiently large.

Similarly one can show that

$$
\sup _{|x|<D_{n+m}}\left|\frac{d}{d x}\left(\sum_{k=2}^{N} b_{n+m}^{(k)} G_{2 k}(x ; \gamma)\right)\right|<\frac{1}{3} \delta_{n+m} .
$$

Now we obtain an estimate of $Q_{n+m}(x)$

$$
\begin{aligned}
& \left\|Q_{n+m}(x)\right\|_{L^{2}\left(\left[-D_{n+m}, D_{n+m}\right]\right)}^{2}<\exp \left(\gamma D_{n+m}^{2}\right) \int_{-D_{n+m}}^{D_{n+m}} Q_{n+m}^{2}(x) \exp \left(-\gamma x^{2}\right) d x \\
& \quad=\exp \left(\gamma D_{n+m}^{2}\right)\left\|Q_{n+m}(x)\right\|_{\mathscr{H}(\gamma)} .
\end{aligned}
$$

It follows from (4.31) that

$$
\exp \left(\frac{1}{2} \gamma D_{n+m}^{2}\right)\left\|Q_{n+m}(x)\right\|_{\mathscr{H}(\gamma)}<\exp (-K),
$$

where

$$
K=(\ln \beta+n)\left(\omega N \ln (2 v)-D^{2} \gamma\right) .
$$

Now we choose the integer $N$ from the condition

$$
\omega N \ln (2 v)-D^{2} \gamma>10 \text {. }
$$

Then

$$
\left\|Q_{n+m}(x)\right\|_{L^{2}\left(\left[-D_{n+m}, D_{n+m}\right]\right)}<\exp (-10(\ln \beta+n))<\delta_{n+m}^{5} .
$$

Further from (4.25) and (4.34)

$$
\begin{aligned}
\sup _{|x|<D_{n+m}}\left|\frac{d}{d x} Q_{n+m}(x)\right| & \leqq \sup _{|x|<D_{n+m}}\left|\frac{d}{d x} q_{n+m}(x)\right|+\sup _{|x|<D_{n+m}}\left|\frac{d}{d x}\left(\sum_{k=2}^{N} b_{n+m}^{(k)} G_{2 k}(x ; \gamma)\right)\right| \\
& +\sup _{|x|<D_{n+m}}\left|\frac{d}{d x}\left(b_{n+m}^{(1)} G_{2}(x ; \gamma)\right)\right| \\
& \leqq 2 \delta_{n}^{0.9}+\frac{1}{3} \delta_{n+m}<\delta_{n}^{0.8}
\end{aligned}
$$


From the Gagliardo-Nirenberg inequality (see [19])

$$
\begin{gathered}
\left\|Q_{n+m}(x)\right\|_{C\left(\left[-D_{n+m}, D_{n+m}\right]\right)} \leqq c_{18}\left\|Q_{n+m}(x)\right\|_{L^{2}\left(\left[-D_{n+m}, D_{n+m}\right]\right)}^{2 / 3} \\
\cdot\left\|\frac{d}{d x} Q_{n+m}(x)\right\|_{C\left(\left[-D_{n+m}, D_{n+m}\right]\right)}<\delta_{n+m}^{2} .
\end{gathered}
$$

Applying the estimate (4.32), (4.33), (4.40) to the relation (4.29) we have that

$$
r_{n+m}(x)=\sum_{k=0}^{1} b_{n+m}^{(k)} G_{2 k}(x ; \gamma)+R_{n+m}^{(1)}(x),
$$

where $\left|R_{n+m}^{(1)}(x)\right|<\delta_{n+m}^{1.5}$. Let us denote

$$
\sum_{k=0}^{1} b_{n+m}^{(k)} G_{2 k}(x ; \gamma)=a_{n+m}^{(0)}+a_{n+m}^{(1)} x^{2},
$$

where

$$
\left|a_{n+m}^{(0)}\right|,\left|a_{n+m}^{(1)}\right|<c_{19}\left(\left|b_{n+m}^{(0)}+b_{n+m}^{(1)}\right|\right)<\delta_{n+m}^{0.9} .
$$

In order to cancel the term $a_{n+m}^{(1)} x^{2}$ in (4.41) we introduce the renormalization of the variable $x$ putting $x=\tilde{x} \sqrt{1+\alpha}$

$$
\begin{aligned}
f_{n+m}(\tilde{x} \sqrt{1+\alpha})= & L_{n+m} \exp \left(-\tilde{x}^{2}-\alpha \tilde{x}^{2}\right)\left(1+r_{n+m}(\tilde{x} \sqrt{1+\alpha})\right) \\
= & L_{n+m} \exp \left(-\tilde{x}^{2}\right)\left(1-\alpha \tilde{x}^{2}+o\left(\left|\alpha \tilde{x}^{2}\right|^{2}\right)+a_{n+m}^{(0)}\right. \\
& \left.+a_{n+m}^{(1)}\left(1+\alpha \tilde{x}^{2}+R_{n+m}^{(1)}(\tilde{x} \sqrt{1+\alpha})\right)\right) .
\end{aligned}
$$

The quantity $\alpha$ is determined by the equation

$$
-\alpha+a_{n+m}^{(1)}=0
$$

in such way that

$$
f_{n+m}(\tilde{x} \sqrt{1+\alpha})=L_{n+m} \exp \left(-\tilde{x}^{2}\right)\left(1+a_{n+m}^{(0)}+R_{n+m}^{(2)}(\tilde{x})\right)
$$

where $\left|R_{n+m}^{(2)}(\tilde{x})\right|<\delta_{n+m}^{1.4}$. Changing now $L_{n+m}$ we eliminate the coefficient $a_{n+m}^{(0)}$. Then

$$
f_{n+m}(\tilde{x} \sqrt{1+\alpha})=\tilde{L}_{n+m} \exp \left(-\tilde{x}^{2}\right)\left(1+\tilde{r}_{n+m}(\tilde{x})\right),
$$

where $\tilde{L}_{n+m}=L_{n+m}\left(1+a_{n+m}^{(0)}\right)$ and for $|\tilde{x}|<D_{n+m}$

$$
\left|\tilde{r}_{n+m}(\tilde{x})\right|=\left|R_{n+m}^{(2)}(\tilde{x})\left(1+a_{n+m}^{(0)}\right)^{-1}\right|<\delta_{n+m} .
$$

Denoting $f_{n+m}(\tilde{x})=f_{n+m}(\tilde{x} \sqrt{1+\alpha})$ we prove the estimate.

The estimates of Lemmas 3.1 and 3.2 for non-typical values of the variable $x$ are an evident consequence of the following statement.

Lemma 4.1. Let for $n=0,1, \ldots$

$$
f_{n+1}(x)=L_{n+1}^{-1}\left(\sum_{t=k \Delta_{n}, k \in \mathbb{Z}^{1}} f_{n}\left(\frac{x}{\sqrt{2 v\left(1+\alpha_{n}\right)}}-t\right) f_{n}\left(\frac{x}{\sqrt{2 v\left(1+\alpha_{n}\right)}}+t\right) \Delta_{n}\right)^{v},
$$


where

and

$$
L_{n+1}=\left(\sum_{t} f_{n}(t) f_{n}(-t) \Delta_{n}\right)^{v}, 0<\alpha_{n}<\delta_{n}^{0.9}, 0<\Delta_{n}<\frac{1}{\sqrt{\beta}}\left(\frac{v}{2}\right)^{0.45 n}
$$

$$
f_{n}(x)=L_{n}^{-1} \exp \left(-x^{2}\right)\left(1+r_{n}(x)\right),\left|r_{n}(x)\right| \leqq \delta_{n}=\frac{1}{\sqrt{\beta}}(2 v)^{-(1-\omega) n}
$$

when $|x|<D_{n}$ and $0<f_{n}(x)<\mathscr{F}_{n}\left(x ; 10 D_{n}\right)$ when $|x| \geqq D_{n}$. Then there exists $\beta_{0}>0$ not depending on $n$ and $v$ such that for any $\beta>\beta_{0}$ and $|x|>D_{n+1}$ :

$$
0<f_{n+1}<\mathscr{F}_{n+1}\left(x ; 10 D_{n+1}\right) .
$$

Proof. Let $\chi(x)$ be a characteristic function of the segment $\left[-10 D_{n}, 10 D_{n}\right]$ and

$$
(f * g){ }^{\operatorname{def}} \sum_{t=k \Delta_{n}, k \in \mathbb{Z}^{1}} f\left(\frac{x}{\sqrt{2 v\left(1+\alpha_{n}\right)}}-t\right) g\left(\frac{x}{\sqrt{2 v\left(1+\alpha_{n}\right)}}+t\right) \Delta_{n} .
$$

Then

$$
\begin{aligned}
f_{n+1} & =L_{n+1}^{-1}\left(f_{n} * f_{n}\right) \\
& =L_{n+1}^{-1}\left(f_{n} \chi * f_{n} \chi+2 f_{n} \chi * f_{n}(1-\chi)+f_{n}(1-\chi) * f_{n}(1-\chi)\right)^{\nu},
\end{aligned}
$$

where $f_{n} \chi(x)=f_{n}(x) \chi(x), f_{n}(1-\chi)(x)=f_{n}(x)(1-\chi(x))$. The function $f_{n}(x)$ satisfies the estimates $\left(A_{n}\right.$ is defined in Sect. 3):

$$
f_{n}(x) \leqq\left\{\begin{array}{l}
\left(1+2 \delta_{n}\right) \exp \left(-x^{2}\right),|x|<D_{n} \\
\frac{1}{2} \exp \left(-0.99 A_{n} x^{2}\right), D_{n} \leqq|x|<10 D_{n} \\
\exp \left(-\frac{1}{2}\left(10 D_{n}\right)^{2}-|x| \ln |x|+10 D_{n} \ln \left(10 D_{n}\right)\right),|x| \geqq 10 D_{n} .
\end{array}\right.
$$

Besides,

$$
L_{n+1}>\left(f_{n} \chi^{(1)} * f_{n} \chi^{(1)}\right)^{v}(0) \geqq\left(\sum_{|t|<D_{n}}\left(1-2 \delta_{n}\right) \exp \left(-2 t^{2}\right) \Delta_{n}\right)^{v}>1,
$$

where $\chi^{(1)}(x)$ is a $\left[-D_{n}, D_{n}\right]$ characteristic function. Now on the basis of the upper bounds on $f_{n}(x)$ we subsequently prove that for $|x| \geqq D_{n+1}$

$$
\begin{aligned}
& f_{n} \chi * f_{n} \chi(x)<\frac{1}{2} \Gamma \equiv \frac{1}{2} \mathscr{F}_{n+1}^{1 / v}\left(x ; 10 D_{n+1}\right), \\
& 2 f_{n} \chi * f_{n}(1-\chi)(x)<0,1 \Gamma, \quad f_{n}(1-\chi) * f_{n}(1-\chi)(x)<0,1 \Gamma .
\end{aligned}
$$

The proof of these estimates is not very difficult but rather long and technical and so we omit it (for the details see [20]). Summing these estimates we prove Lemma 4.1. Thus the proof Lemmas 3.1 and 3.2 is over.

\section{Initial Conditions}

We now prove Lemma 3.3 for the initial function $F_{0}(j ; \beta)$. For brevity in this section we shall write $\beta$ instead of $\beta / m$ so that according to (1.8) we have

$$
F_{0}(j ; \beta)=K_{0} \int_{-\pi}^{\pi} \exp (\beta \cos \alpha+i j \alpha) d \alpha,
$$


where $K_{0}=\left[\int \exp (\beta \cos \alpha) d \alpha\right]^{-1}$. It is obvious that such a change is unessential because the Lemma 3.3 is formulated only for sufficiently large values of $\beta$. Given $\alpha=t \beta^{-1 / 2}$ we easily deduce the following expression for the initial function

$$
\begin{aligned}
F_{0}(j ; \beta)= & \tilde{K}_{0} \exp \left(-\frac{j^{2}}{2 \beta}\right) \int_{-\pi \sqrt{\beta}}^{\pi \sqrt{\beta}} \exp \left(-\left(\frac{i j}{\sqrt{2 \beta}}-\frac{t}{\sqrt{2}}\right)^{2}\right) \\
& \cdot \exp \left(\beta\left(\cos \frac{t}{\sqrt{\beta}}-1+\frac{t^{2}}{2 \beta}\right)\right) d t
\end{aligned}
$$

where $\tilde{K}_{0}=K_{0} \beta^{-1 / 2} \exp \beta$. Assume that

$$
\begin{aligned}
1+\tilde{r}_{0}\left(\gamma_{0} j ; \beta\right)= & \int_{|t| \leqq \pi \sqrt{\beta}} \exp \left(-\left(\frac{i j}{\sqrt{2 \beta}}-\frac{t}{\sqrt{2}}\right)^{2}\right) \\
& \cdot \exp \left(\beta\left(\cos \frac{t}{\sqrt{\beta}}-1+\frac{t^{2}}{2 \beta}\right)\right) d t,
\end{aligned}
$$

where $\gamma_{0}=(2 \beta)^{-1 / 2}$. Then from (5.2) we obtain that

$$
F_{0}(j ; \beta)=\tilde{K}_{0} \exp \left(-\left(\gamma_{0} j\right)^{2}\right)\left(1+\tilde{r}_{0}\left(\gamma_{0} j ; \beta\right)\right) .
$$

From the definition of the function $f(x)$ putting $x=\gamma_{0} j$ we have that

$$
\begin{aligned}
f_{0}(x ; \beta)= & \tilde{K}_{0} \exp \left(-x^{2}\right) \int_{|t| \leqq \pi \sqrt{\beta}} \exp \left(-\left(i x-\frac{t}{\sqrt{2}}\right)^{2}\right) \\
& \cdot \exp \left(\beta\left(\cos \frac{t}{\sqrt{\beta}}-1+\frac{t^{2}}{2 \beta}\right)\right) d t
\end{aligned}
$$

Now we represent the function $1+\tilde{r}_{0}(x ; \beta)$ as a sum of two integrals

$$
\begin{aligned}
1+\tilde{r}_{0}(x ; \beta)= & \int_{|t| \leqq \beta^{1 / 8}} \exp \left(-\left(i x-\frac{t}{\sqrt{2}}\right)^{2}\left(1+\frac{t^{4}}{24 \beta}+R_{1}(t ; \beta)\right) d t\right) \\
& +\int_{\beta^{1 / 8}<|t| \leqq \pi V \bar{\beta}} \exp \left(-\left(i x-\frac{t}{\sqrt{2}}\right)^{2}\right) \\
& \cdot \exp \left(\beta\left(\cos \frac{t}{\sqrt{\beta}}-1+\frac{t^{2}}{2 \beta}\right)\right) d t .
\end{aligned}
$$

Here $R_{1}(t ; \beta)$ is the remainder term in the expansion of the function $\exp \left(\beta\left(\cos \frac{t}{\sqrt{\beta}}-1+\frac{t^{2}}{2 \beta}\right)\right)$ and it's evident that $\left|R_{1}(t ; \beta)\right| \leqq c \frac{t^{6}}{\beta^{2}}$ where $c$ is a positive constant. Hence it is not difficult to obtain that

$$
1+\tilde{r}_{0}(x ; \beta)=\sqrt{2 \pi}\left(1+S_{1}(x ; \beta)+S_{2}(x ; \beta)+S_{3}(x ; \beta)+S_{4}(x ; \beta)\right),
$$


where

$$
\begin{aligned}
S_{1}(x ; \beta)= & \frac{1}{\sqrt{2 \pi}} \int_{|t|>\beta^{1 / 8}} \exp \left(-\left(i x-\frac{t}{\sqrt{2}}\right)^{2}\right) d t \\
S_{2}(x ; \beta)= & \frac{1}{\sqrt{2 \pi}} \int_{|t| \leqq \beta^{1 / 8}} \exp \left(-\left(i x-\frac{t}{\sqrt{2}}\right)^{2}\right) \frac{t^{4}}{24 \beta} d t, \\
S_{3}(x ; \beta)= & \frac{1}{\sqrt{2 \pi}} \int_{|t| \leqq \beta^{1 / 8}} \exp \left(-\left(i x-\frac{t}{\sqrt{2}}\right)^{2}\right) R_{1}(t ; \beta) d t, \\
S_{4}(x ; \beta)= & \frac{1}{\sqrt{2 \pi}} \int_{\beta^{1 / 8}<|t| \leqq \pi \sqrt{\beta}} \exp \left(-\left(i x-\frac{t}{\sqrt{2}}\right)^{2}\right) \\
& \cdot \exp \left(\beta\left(\cos \frac{t}{\sqrt{\beta}}-1+\frac{t^{2}}{2 \beta}\right)\right) d t .
\end{aligned}
$$

Now from (5.3) it follows that

$$
f_{0}(x ; \beta)=L_{0} \exp \left(-x^{2}\right)\left(1+r_{0}(x ; \beta)\right)
$$

where $L_{0}=\sqrt{2 \pi} \tilde{K}_{0}$ and $r_{0}(x ; \beta)=S_{1}(x ; \beta)+S_{2}(x ; \beta)+S_{3}(x ; \beta)+S_{4}(x ; \beta)$. We shall investigate each integral $S_{1}(x ; \beta), \ldots, S_{4}(x ; \beta)$ separately for $|x|<D_{0}$ verify the validity of the estimate of the condition $\mathbf{V}^{(0)}$.

In view of

$$
\int_{t}^{\infty} \exp \left(-u^{2}\right) d u \sim t^{-1} \exp \left(-t^{2}\right), \text { when } t \rightarrow \infty
$$

we can directly estimate $S_{1}(x ; \beta)$ for sufficiently large $\beta$ :

$$
\left|S_{1}(x ; \beta)\right| \leqq \frac{1}{\sqrt{2 \pi}} \exp \left(D^{2} \ln \beta\right) \int_{|t|>\beta^{1 / 8}} \exp \left(-\frac{t^{2}}{2}\right) d t<\frac{1}{4} \beta^{-1 / 2} .
$$

Further let us note that

$$
4 \int_{-\infty}^{\infty} \exp \left(-\left(i x-\frac{t}{\sqrt{2}}\right)^{2}\right) t^{4} d t=\exp x^{2} \frac{d^{4}}{d x^{4}} \exp \left(-x^{2}\right)=H_{4}(x)
$$

so for the second term we have

$$
\left|S_{2}(x ; \beta)\right| \leqq \frac{1}{\sqrt{2 \pi}}\left|\int_{|t|>\beta^{1 / 8}} \frac{t^{4}}{24 \beta} \exp \left(-\left(i x-\frac{t}{\sqrt{2}}\right)^{2}\right) d t\right|+\text { const }\left|\frac{H_{4}(x)}{\beta}\right|
$$

and next due to the relations

$$
\left|H_{4}(x)\right| \leqq \text { const } \ln ^{2} \beta ; \int_{t}^{\infty} u^{4} \exp \left(-u^{2}\right) d u \sim t^{3} \exp \left(-t^{2}\right), \text { when } t \rightarrow \infty
$$


for sufficiently large $\beta$ we obtain the desired estimate

$$
\left|S_{2}(x ; \beta)\right|<\frac{1}{4} \beta^{-1 / 2} .
$$

Now using the analyticity of $R_{1}(t ; \beta)$ and the change of variables $u=\frac{t}{\sqrt{2}}-i x$ we estimate $S_{3}(x ; \beta)$. By Cauchy theorem it follows that

$$
\begin{aligned}
\left|S_{3}(x ; \beta)\right|= & \frac{1}{\sqrt{2 \pi}} \mid\left(\int_{-\beta^{1 / 8}}^{-\beta^{1 / 8}+i x \sqrt{2}}+\int_{-\beta^{1 / 8}+i x \sqrt{2}}^{\beta^{1 / 8}+i x \sqrt{2}}+\int_{\beta^{1 / 8}+i x \sqrt{2}}^{\beta^{1 / 8}}\right) \\
& \cdot \exp \left(-u^{2}\right) R_{1}(\sqrt{2}(u+i x) ; \beta) d u \mid .
\end{aligned}
$$

It is evident that the following inequality is valid in the domain of integration

$$
\left|R_{1}(t ; \beta)\right| \leqq c \frac{|t|^{6}}{\beta^{2}} \leqq c_{1} \frac{\left(|x \sqrt{2}|^{2}+\left(\beta^{1 / 8}\right)^{2}\right)^{3}}{\beta^{2}}=o\left(\beta^{-1}\right),
$$

where $c, c_{1}$ are some positive constants. Therefore

$$
\left|S_{3}(x ; \beta)\right| \leqq \frac{\text { const }}{\beta} \int_{|u|<\beta^{1 / 8}} \exp \left(-u^{2}\right) d u+o\left(\exp \left(2 x-\beta^{1 / 4}\right)\right) \leqq \frac{1}{4} \beta^{-1 / 2} .
$$

Set $t=\alpha \sqrt{\beta}$ and rewrite $S_{4}(x ; \beta)$ in the following form

$$
S_{4}(x ; \beta)=\frac{1}{\sqrt{2 \pi}} \exp x^{2} \int_{\pi \beta^{-3 / 8}<|\alpha|<\pi} \exp (-i \alpha x \sqrt{2 \beta}+\beta(\cos \alpha-1)) d \alpha .
$$

Then from the inequality $\cos \pi \beta^{-3 / 8}<1-\frac{1}{3} \pi \beta^{-3 / 4}$ we have for the last integral that

$$
\left|S_{4}(x ; \beta)\right| \leqq \frac{1}{\sqrt{2 \pi}} \exp \left(D^{2} \ln \beta\right) \exp \left(\beta\left(-\frac{\pi}{3 \beta^{3 / 4}}\right)\right) \int_{\pi \beta^{-3 / 8}<|\alpha|<\pi} d \alpha<\frac{1}{4} \beta^{-1 / 2} .
$$

From (5.4) and estimates (5.5)-(5.8) it follows the inequality $v_{1}$ ) of the condition $\mathbf{V}^{(0)}$.

To prove the inequality $\mathrm{v}_{2}$ ) of the condition $\mathbf{V}^{(0)}$ we consider separately the cases when $D_{0} \leqq|x|<10 D_{0}$ and $|x| \geqq 10 D_{0}$. In the first case we use the fact that for $|x|<10 D_{0}$

$$
f_{0}(x)<\left(1+o\left(\beta^{-1 / 2}\right)\right) \exp \left(-x^{2}\right)
$$

which is a consequence of the above considerations. In view of the fact that

$$
\exp \left(-x^{2}\right)<\exp \left(-0.99 x^{2}\right) \exp \left(-0.01 D_{0}^{2}\right)
$$

for $|x| \geqq D_{0}$ and $\lim _{\beta \rightarrow \infty} \exp \left(-0.01 D_{0}^{2}\right)=0$ we have

$$
f_{0}(x)<\frac{1}{2} \exp \left(-0.99 x^{2}\right)
$$

if $\beta$ is large enough, which is what we wanted to prove. 
To consider the second case we use Captain's inequality for modified Bessel functions (see, e.g. [12]):

$$
I_{j}(\beta) \leqq \frac{\left(\frac{\beta}{j}\right)^{j} \exp \left(j \sqrt{1+\left(\frac{\beta}{j}\right)^{2}}\right)}{\left(1+\sqrt{\left.1+\left(\frac{\beta}{j}\right)^{2}\right)^{j}}\right.} .
$$

Since $I_{0}(\beta)>\beta^{-1 / 2} \exp \beta$ we can obtain for the initial function of our model, that

$$
F_{0}(j ; \beta) \leqq \frac{\sqrt{\beta}\left(\frac{\beta}{j}\right)^{j} \exp \left(j \sqrt{1+\left(\frac{\beta}{j}\right)^{2}}\right)}{\exp \beta\left(1+\sqrt{\left.1+\left(\frac{\beta}{j}\right)^{2}\right)^{j}}\right.}
$$

Let us put $x=j(2 \beta)^{-1 / 2}$ and denote $z=\frac{\beta}{j}=\frac{1}{x} \sqrt{\frac{\beta}{2}}$. In the sequel we shall consider only the case $x \geqq 10 D_{0}\left(\right.$ corr. $\left.z<z_{0}=\frac{1}{10 D_{0}} \sqrt{\frac{\beta}{2}}\right), D_{0}=D \ln \beta$. The proof when $x \leqq-10 D_{0}$ is analogous. Using (5.10) for the function $f_{0}(x ; \beta)$ we obtain the inequality

$$
f_{0}(x ; \beta) \leqq \sqrt{\beta} \exp \left(-\left(\varphi_{1}(z)+\varphi_{2}(z)\right) x^{2}\right),
$$

where

$$
\left\{\begin{array}{l}
\varphi_{1}(z)=2 z^{2}-2 z \sqrt{1+z^{2}} \\
\varphi_{2}(z)=-2 z \ln \frac{z}{1+\sqrt{1+z^{2}}} .
\end{array}\right.
$$

The following properties of the functions just introduced can be easily verified:

1) $\varphi_{1}(z), \varphi_{2}(z)$ are bounded smooth functions for $z>0$;

2) $\varphi_{1}(0)=0, \varphi_{1}(\infty)=-1$

3) $\varphi_{2}(\infty)=2, \varphi_{2}(z) \sim-2 z \ln z$, when $z \rightarrow+0$.

Let us denote

$$
\begin{aligned}
& \varphi(z)=\varphi_{1}(z)+\varphi_{2}(z),(\varphi(\infty)=1, \varphi(z) \sim 2 z|\ln z|, \text { when } z \rightarrow+0), \\
& \Phi(x)=\varphi(z) x^{2}, \Psi(x)=\left(10 D_{0}\right)^{2}+x \ln x-10 D_{0} \ln \left(10 D_{0}\right) .
\end{aligned}
$$

Now it remains to prove that

$$
\Phi(x)>\Psi(x) \text {, when } x \geqq 10 D_{0} .
$$

It follows directly from

Lemma 5.1. For $x \geqq 10 D_{0}$ the following assertions are valid
1. $\Phi\left(10 D_{0}\right)>\Psi\left(10 D_{0}\right)$.
2. $\Phi^{\prime}(x)>\Psi^{\prime}(x)$. 
Proof. 1. From (5.12) we have

$$
\Psi\left(10 D_{0}\right)=\left(10 D_{0}\right)^{2} ; \quad \Phi\left(10 D_{0}\right)=\varphi\left(z_{0}\right)\left(10 D_{0}\right)^{2} .
$$

Moreover from the definition of the function $\varphi(z)$ it follows that $\varphi\left(z_{0}\right)>1$ if $z_{0}>1$.

2. In view of

$$
\begin{aligned}
& \Phi_{x}^{\prime}(z)=\sqrt{\frac{\beta}{2}}\left(2 \frac{\varphi(z)}{z}-\varphi^{\prime}(z)\right)=-\sqrt{2 \beta} \ln \frac{z}{1+\sqrt{1+z^{2}}} ; \\
& \Psi_{x}^{\prime}(z)=\left(\ln \sqrt{\frac{\beta}{2}}+1\right)-\ln z
\end{aligned}
$$

we need to prove that for $z<z_{0}$

$$
F(z)=\Phi_{x}^{\prime}(z)-\Psi_{x}^{\prime}(z)>0 .
$$

The last inequality follows directly from the validity of the next assertions :
A. $F\left(z_{0}\right)>0$.
B. $-F^{\prime}(z)>0$, when $z<z_{0}$.

The first assertion $\mathbf{A}$ is evident ( $\beta$ can be choosed a sufficiently large) and the second one can be obtained in the following way. From the relation

$$
-F^{\prime}(z)=\sqrt{2 \beta}\left(\frac{1}{z}-\frac{z}{\left(1+\sqrt{1+z^{2}}\right) \sqrt{1+z^{2}}}\right)-\frac{1}{z}
$$

on can see that $\mathbf{B}$ is valid if $1-(2 \beta)^{-1 / 2}>1-\left(1+z^{2}\right)^{-1 / 2}$. As $z<z_{0}=\frac{1}{10 D} \sqrt{\frac{\beta}{2 \ln \beta}}$, the last inequality is obviously true for sufficiently large $\beta$.

Using Lemma 5.1 and according to (5.12), (5.13) we obtain from (5.11) the following inequality:

$$
f_{0}(x ; \beta) \leqq \sqrt{\beta} \exp \left(-\frac{1}{2}\left(10 D_{0}\right)^{2}\right) \exp \left(-\frac{1}{2}\left(10 D_{0}\right)^{2}-x \ln x+10 D_{0} \ln \left(10 D_{0}\right)\right) .
$$

Now due to the evident estimate $\sqrt{\beta} \exp \left(-50 D_{0}^{2}\right)<1$ for sufficiently large $\beta$ we obtain the assertion $\mathrm{v}_{2}$ ) of the condition $\mathbf{V}^{(0)}$.

Acknowledgments. The authors express their gratitude to Professors A. A. Migdal, Ja. G. Sinai for valuable discussions and Dr. D. Surgailis for useful remarks. We thank very much to Professor G. Jona-Lasinio for his help in preparing this text for publication.

\section{References}

1. Wilson, K.G., Kogut, I.: Phys. Rep. 12 C, 75-199 (1974)

2. Wilson, K.G.: Rev. Mod. Phys. 47, 773 (1975)

3. Kadanoff, L.P.: Notes on Migdal's recursion formulas. Preprint, IBM Research Lab., Zürich, Switzerland (1976)

4. Patashinskij, A.Z., Pokrovskij, V.L.: Usp. Phys. Nauk. 121, 55 (1977) (in Russian)

5. Migdal, A.A.: JETP 69, 810 (1975) (in Russian)

6. Migdal, A.A. : JETP 69, 1457 (1975) (in Russian)

7. Bleher, P.M., Sinai, Ja.G.: Commun. Math. Phys. 33, 23-42 (1973)

8. Bleher, P.M., Sinai, Ja.G.: Commun. Math. Phys. 45, 247 (1975)

9. Bleher, P.M.: Tr. Mosc. Math. Ob. 33, 155 (1975) (in Russian) 
10. Bleher, P.M.: Usp. Math. Nauk. 32, 243 (1977) (in Russian)

11. Sinai, Ja.G.: Mathematical problems of the theory of phase transitions. Hungary (1979) (in press)

12. Watson, G.N.: A treatise on the theory of Bessel functions. New York: Repr. Cambridge Univ. Press; Macmillan 1945

13. Bateman, G., Erdelyi, A.: Higher trancendental functions, Vol. 2. New York, Toronto, London: McGraw-Hill 1955

14. Ginibre, J.: Commun. Math. Phys. 16, 310 (1970)

15. Bleher, P.M.: The numerical solution of the approximate equations of Migdal's renormgroup. Preprint, Inst. Appl. Math., USSR Academy of Sciences, No. 48 (1976) (in Russian)

16. Asano, T.: Phys. Rev. Letters 24, 1409 (1970)

17. Householder, A.S. : Principles of numerical analysis. New York, Toronto, London: McGraw-Hill 1953

18. Sarkar, S.: Phys. Rev. S $16(8), 2666$ (1977)

19. Besov, O.V., Nikolskij, S.M., Iljin, V.P.: Integral representations of functions and imbedding theorems. Moscow: Nauka 1975 (in Russian)

20. Bleher, P.M., Žalys, E.: The investigation of low- and high-temperature fixed points in the Migdal's equations. Preprint, Inst. Appl. Math., USSR Academy of Sciences (1978) (in Russian)

Communicated by E. Lieb

Received September 13, 1978 